\title{
АЛГОРИТМ АВТОМАТИЗИРОВАННОГО УПРАВЛЕНИЯ ПРОЦЕССОМ ДЕСУБЛИМАЦИИ ПРОИЗВОДСТВА ГЕКСАФТОРИДА УРАНА
}

\author{
Николаев Александр Валерьевич', \\ niav@tpu.ru
}

Криницын Николай Станиславович',
kns@tpu.ru

Дядик Валерий Феодосиевич', dyadik@tpu.ru
' Национальный исследовательский Томский политехнический университет, Россия, 635050, г. Томск, пр. Ленина, 30.

\begin{abstract}
Актуальность исследования обусловлена требованием к равномерному заполнению товарным гексафторидом урана транспортных емкостей и принятием решения о прекращении наполнения ёмкости по степени её объёмного заполнения. Указанное требование может быть достигнуто вводом в эксплуатацию автоматизированной системы управления аппаратом десублимации. Цель: разработка алгоритма расчёта интервала захолаживания трубчатки аппарата десублимации, обеспечивающего накопление заданной толщины слоя десублимата в автоматизированном режиме.

объект: аппарат десублимации производства гексафторида урана.

Методы: математическое моделирование технологических процессов; проведение полных факторных экспериментов на математической модели; регрессионный анализ; статистический анализ; производственные эксперименты на аппарате десублимации производства ГФУ.

Результаты. Для реализации системы стабилизации заданной толщины слоя десублимата на охлаждаемом элементе в качестве управляющего воздействия используется длительность интервала захолаживания. Для проведения вычислительных экспериментов и нахождения аналитической зависимости расчёта длительности интервала захолаживания используется математическая модель аппарата десублимации, описывающая протекающие в нём теплообменные и массообменные процессы. Адекватность модели подтверждена сравнением результатов математического моделирования и производственных данных. Вывод об адекватности модели сделан по величине приведённой среднеквадратичной погрешности предсказания моделью производственных данных. В результате полного факторного эксперимента на разработанной математической модели получено уравнение расчёта интервала захолаживания. Уравнение позволяет исходя из значений регистрируемых на производстве технологических переменных рассчитывать необходимую длительность интервала захолаживания трубчатки, в течение которого будет накоплен заданный оператором слой десублимата.
\end{abstract}

Ключевые слова:

Десублимация, гексафторид урана, теплообмен, массоперенос, математическое моделирование.

\section{Введение}

Производство гексафторида урана (ГФУ) является востребованным в России на сегодняшний день. Активная программа Госкорпорации Росатом по развитию и строительству новых энергоблоков АЭС ежегодно увеличивает количество потребителей данного продукта [1]. Сублиматный завод $\mathrm{AO}$ «Сибирский химический комбинат» является единственным производителем ГФУ в России. Это накладывает высокие требования к стабильности работы предприятия и качеству выпускаемой продукции.

Непрерывная работа предприятия в целом и узла десублимации в частности обеспечивает выполнение поставленных планов перед заводом по выработке ГФУ. Одним из факторов, позволяющих оперативно изменять производительность аппарата десублимации, является варьирование длительности интервала захолаживания [2]. За счёт этого изменяется толщина слоя накапливаемого десублимата, а следовательно, и масса сбрасываемого продукта в единицу времени. Однако неконтролируемое увеличение длительности интервала захолаживания может привести к накоплению критической массы, в результате сброса которой произойдет забивание высыпной горловины, и выходу аппарата из строя. С другой стороны, слишком частые сбросы десублимата приведут к снижению эффективности аппарата и высокому проскоку сырья. Помимо длительности захолаживания на толщину накапливаемого слоя влияет вид и состав перерабатываемого сырья. В зависимости от доли тетрафторида урана или октаоксида триурана в составе загружаемого сырья концентрация ГФУ в составе технологического газа изменяется от 30 до 70 об. \% .

Показателем качества выпускаемой продукции является значение насыпной плотности. Она характеризуется плотностью накапливаемого слоя десублимата и, как следствие, конечной массой заполняемых транспортных ёмкостей. Насыпная плотность зависит от длительности захолаживания, температуры контактной поверхности между технологическим газом и наросшим десублиматом, а также от расхода технологического газа и концентрации ГФУ в его составе. Рациональным способом стабилизации плотности ГФУ в условиях производства является тепловой сброс продукта в момент накопления слоя десублимата фиксиро- 
ванной толщины, что обеспечит стабильность производительности аппарата и минимизацию тепловых переходных процессов.

\section{Конструкция аппарата}

Для осуществления процесса десублимации в промышленности применяются теплообменные аппараты различных конструкций $[3,4]$. На Сибирском химическом комбинате используется кожухотрубный теплообменник (рис. 1), представляющий собой вертикальный обогреваемый цилиндрический аппарат. Внутри аппарата смонтирована трубчатка из трубок Фильдо, разделенная на секции.

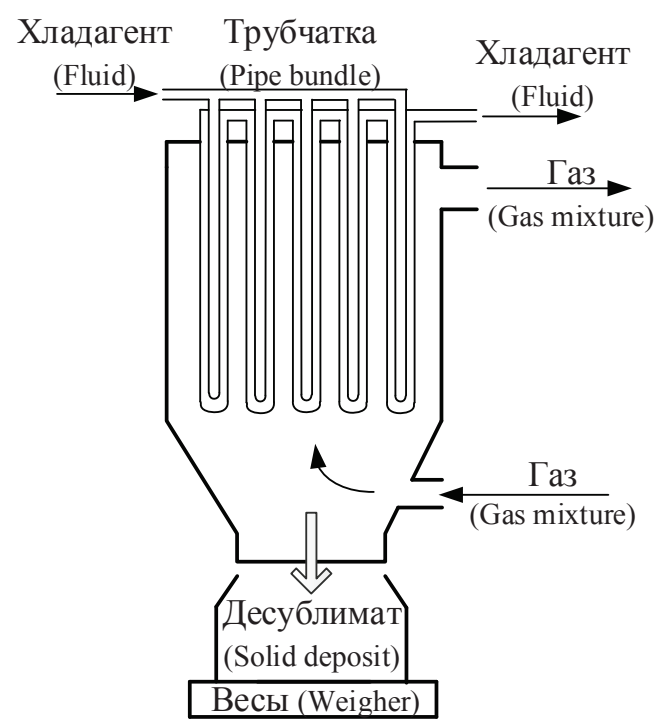

Puc. 1. Конструкция аппарата десублимации

Fig. 1. Construction of deposition apparatus

Работа аппарата десублимации предусматривает два режима: накопление десублимата на внешней поверхности трубчатки и его сброс. Накопление обеспечивается подачей хладагента в трубчатку аппарата. В результате охлаждения трубчатки и технологического газа на поверхности нарастает слой десублимата. Режим сброса характеризуется подачей в трубчатку аппарата теплоносителя. За счёт нагревания поверхности на границе раздела трубчатки и десублимата происходит сублимация пограничного слоя. Под действием силы тяжести корки десублимата осыпаются в транспортную емкость, находящуюся под аппаратом.

Накопление твёрдого ГФУ в транспортной емкости происходит вплоть до её заполнения до фиксированного уровня, что обеспечивает постоянство объема в ней. Масса заполняемых транспортных емкостей отслеживается с помощью весоизмерительного устройства. На рис. 2 представлен график, отражающий динамику заполнения двух транспортных емкостей сбрасываемым десублиматом. Итоговая масса емкостей различается на $3 \%$, что выходит за пределы установленного на заводе допуска в $1 \%$.

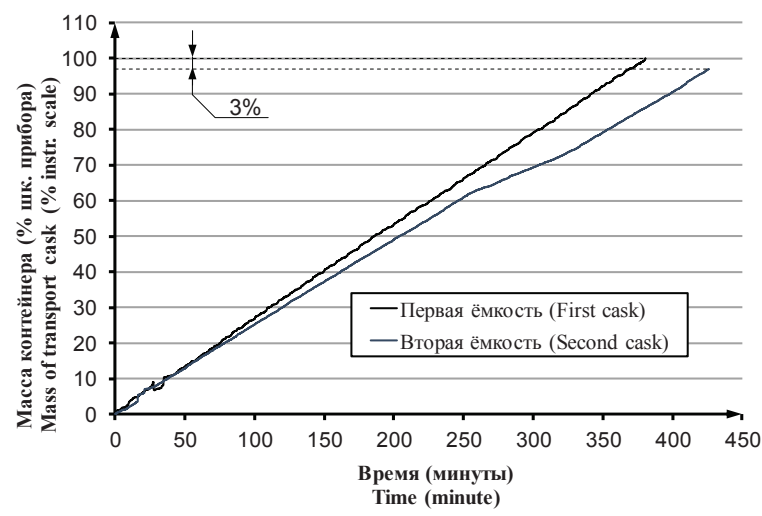

Рис. 2. График заполнения двух транспортных елкостей

Fig. 2. Graph of loading of two transport casks

В настоящее время на Сублиматном заводе АО «СХК» реализована система управления аппаратами узла десублимации с фиксированным интервалом захолаживания. На вход аппарата десублимации поступает технологический газ, состав и расход которого изменяются в зависимости от состава перерабатываемого сырья. Как результат, доля ГФУ в составе технологического газа меняется от 30 до 70 об. \%. Принимая во внимание фиксированный интервал захолаживания трубчатки аппарата десублимации, никак не зависящий от состава технологического газа на входе в аппарат, можно говорить о накоплении на поверхности охлаждаемых элементов слоя десублимата различной толщины. Это является причиной нестабильности насыпной плотности готового продукта в транспортных емкостях.

Для прогнозирования величины насыпной плотности готового продукта и производительности аппарата десублимации предлагается реализовать систему стабилизации заданной толщины слоя десублимата. Для этого при расчёте интервала захолаживания следует учитывать влияющие на процесс накопления десублимата регистрируемые на производстве технологические переменные: расход и состав технологического газа на входе аппарата, температуру хладагента в трубчатке аппарата $[5,6]$.

Для проведения вычислительных экспериментов с целью получения регрессионного уравнения, позволяющего рассчитывать интервал захолаживания трубчатки десублиматора, используется модернизированная математическая модель аппарата десублимации производства гексафторида урана [7]. При модернизации модели добавлено уравнение, учитывающее изменение давления технологического газа внутри аппарата. Это позволило рассчитать текущую скорость передвижения технологического газа, на которую влияют тепловые и масообменные процессы, происходящие внутри аппарата. Для математического моделирования работы действующего на производстве аппарата десублимации в модели учтены реальные геометрические размеры и режимы его работы. 


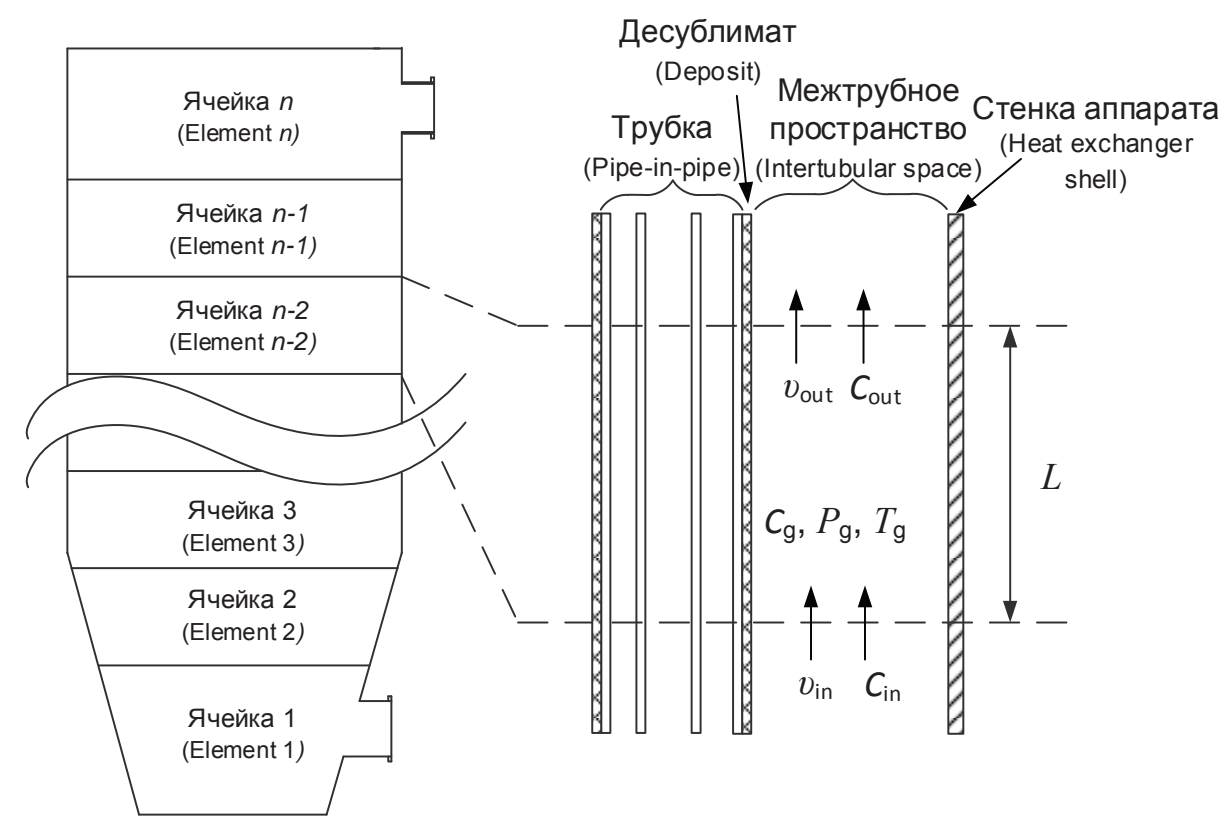

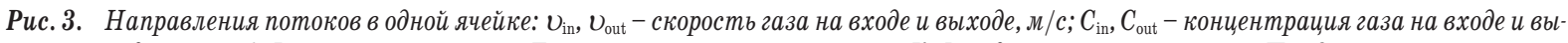

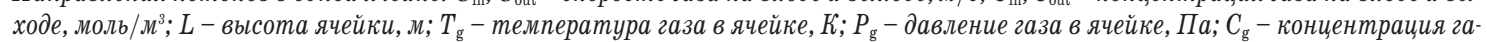
за в ячейке, моль $/ \mathrm{m}^{3}$

Fig. 3. Gas mixture flow directions in an element: $v_{\text {in }}, v_{\text {out }}$ are the inlet and outlet gas mixture velocity, respectively, $m / s ; C_{\text {in }}, C_{\text {out }}$ are the inlet and outlet concentration, respectively, mole $/ \mathrm{m}^{3} ; L$ is the element height, $m ; T_{\mathrm{g}}$ is the gas mixture temperature, $K$; $P$ is the gas mixture pressure, $\mathrm{Pa} ; \mathrm{C}_{\mathrm{g}}$ is the gas mixture concentration, mole $/ \mathrm{m}^{3}$

\section{Математическая модель аппарата десублимации}

Движение газа

Математическая модель десублиматора построена с использование ячеечной структуры [8]. Применение такой структуры подразумевает разбиения пространства на поперечные ячейки по высоте аппарата. Моделирование заключается в последовательном расчёте параметров всех ячеек по выбранному направлению. Направление расчёта соответствует направлению движения технологического газа в межтрубном пространстве аппарата: снизу вверх. На рис. 3 представлены направления потоков технологического газа в одной ячейке высотой $L$.

В ячеечной модели аппарата десублимации приняты следующие допущения:

- расчет распределения компонентов технологического газа только по одной пространственной координате (по высоте десублиматора);

- пренебрежение распределением потока и температуры технологического газа по радиусу аппарата;

- фазовому переходу подвергается только $\mathrm{UF}_{6}$;

- скорость движения и температура хладагента по высоте аппарата во всех секциях одинаковы;

- плотность десублимата постоянна;

- десублимат равномерно распределяется между трубками, входящими в одну секцию;

- переходные процессы при смене теплоносителя в трубчатке происходят мгновенно.

Каждая ячейка десублиматора описывает состояние технологического газа в межтрубном про- странстве и представляет собой модель идеального смешения, то есть характеризуется полным и мгновенным перемешиванием вновь поступающего вещества с уже находящимся в ячейки. Учитывая состав технологического газа $\left(\mathrm{UF}_{6}, \mathrm{HF}, \mathrm{O}_{2}, \mathrm{~N}_{2}\right.$, $\mathrm{F}_{2}$ ), процесс изменения концентраций веществ внутри ячейки описывается законом сохранения массы в виде системы уравнения:

$$
\left\{\begin{array}{l}
\frac{d C_{\mathrm{UF}_{6}}}{d t}=\frac{1}{V_{\text {elt }}}\left(v_{\text {in }} A_{\text {in }} C_{\mathrm{UF}_{6} \text { in }}-v_{\text {out }} A_{\text {out }} C_{\mathrm{UF}_{6} \text { out }}\right)-\frac{W_{\mathrm{UF}_{6}}}{V_{\text {elt }}} ; \\
\frac{d C_{y}}{d t}=\frac{1}{V_{\text {elt }}}\left(v_{\text {in }} A_{\text {in }} C_{y \text { in }}-v_{\text {out }} A_{\text {out }} C_{y \text { out }}\right),
\end{array}\right.
$$

где $y$ - компонент технологического газа $\mathrm{HF}, \mathrm{O}_{2}$, $\mathrm{N}_{2}, \mathrm{~F}_{2} ; W_{\text {UF6 }} / V_{\text {elt }}$ описывает явление десублимации $\mathrm{UF}_{6} ; A_{\text {in }}, A_{\text {out }}$ - площадь поперечного сечения ячейки, $\mathrm{M}^{2} ; V_{\text {elt }}$ - объём ячейки, $\mathrm{M}^{3} ; W_{\text {UF6 }}-$ скорость десублимации.

Текущая концентрация смеси компонентов технологического газа рассчитывается из уравнения Менделеева-Клайперона:

$$
\frac{d C_{\mathrm{g}}}{d t}=\frac{1}{R T_{\mathrm{g}}} \frac{d P_{\mathrm{g}}}{d t},
$$

где $C_{\mathrm{g}}=C_{\mathrm{UF} 6}+C_{\mathrm{HF}}+C_{02}+C_{\mathrm{N} 2}+C_{\mathrm{F} 2}-$ концентрация газовой смеси, моль $/ \mathrm{M}^{3} ; R$ - газовая постоянная, Дж/моль $\cdot \mathrm{K}$;

Изменение давления в ячейки за счёт проходящих тепловых и массообменных процессов рассчитывается как суперпозиция всех его составляющих: 


$$
\frac{d P_{\mathrm{g}}}{d t}=R T_{\text {in }} v_{\text {in }} \frac{A_{\text {in }}}{V_{\text {elt }}} C_{\text {in }}-R T_{\mathrm{g}} \frac{W_{\mathrm{UF}_{6}}}{V_{\text {elt }}}+R C_{\mathrm{g}} \frac{d T_{\mathrm{g}}}{d t},
$$

где $T_{\text {in }}$ - температура газа на входе, $\mathrm{K}$.

Каждое слагаемое описывает следующее взаимодействие: $R T_{\text {in }} v_{\text {in }} \frac{A_{\text {in }}}{V_{\text {elt }}} C_{\text {in }}-$ изменение давление за счёт движения потока технологического газа; $R T_{\mathrm{g}} \frac{W_{\mathrm{UF}_{6}}}{V_{\text {elt }}}-$ изменение давление за счёт массообменных процессов; $R C_{\mathrm{g}} \frac{d T_{\mathrm{g}}}{d t}-$ изменение давления за счёт тепловых процессов.

Для расчёта температуры технологического газа и массы накапливаемого десублимата на трубчатке аппарата в рамках одной ячейки используются уравнения, учитывающие теплообменные и массообменные процессы, протекающие внутри ячейки.

В связи с последовательным и циклическим режимом работы секций трубчатки аппарата десублимации выполняется моделирование накопления и сброса десублимата посекционно. Это подразумевает, что для каждой из секции ведётся расчёт следующих составляющих в рамках каждой ячейки:

- температура накопленного десублимата;

- масса наросшего слоя десублимата.

\section{Тепловые взаимодействия внутри ячейки}

Направление движения тепловых потоков в рамках одной ячейки представлено на рис. 4 .

Выражения для расчёта температуры технологического газа и температуры десублимата для $i$ секции в рамках одной ячейки получены из формулы расчёта количества теплоты, передаваемого при нагревании и охлаждении:

$$
\frac{d T_{\mathrm{g}}}{d t}=\frac{1}{c_{\mathrm{g}} m_{\mathrm{g}}} \frac{Q_{\mathrm{g}}}{d t} ; \quad \frac{d T_{\mathrm{ds} i}}{d t}=\frac{1}{c_{\mathrm{ds}} m_{\mathrm{ds} i}} \frac{Q_{\mathrm{ds} i}}{d t},
$$

где $i$ - номер секции; $T_{\mathrm{ds}}$ - температура десублимата, $\mathrm{K} ; T_{\mathrm{g}}$ - температура технологического газа, $\mathrm{K}$; $c_{\mathrm{g}}, c_{\mathrm{ds}}$ - теплоёмкость технологического газа и десублимата соответственно, Дж/кг/K; $m_{\mathrm{g}}, m_{\mathrm{ds}}$ - масса технологического газа и десублимата соответственно, кг.

Количество тепла, передаваемое технологическому газу в ячейке, вычисляется как суперпозиция всех источников тепла, взаимодействующих с ним:

$$
Q_{\mathrm{g}}=Q_{\text {shell }}+Q_{\text {in }}+\sum_{i=1}^{9} Q_{\mathrm{pt} i} .
$$

Количество тепла, передаваемое слою десублимата, отложившемуся на поверхности одной секции в ячейки, вычисляется как суперпозиция всех источников тепла, взаимодействующих с ним:

$$
Q_{\mathrm{ds} i}=Q_{\mathrm{g}}+Q_{\mathrm{pt} i}+Q_{\text {fluid } i} .
$$

Количество тепла, передаваемое от стенки аппарата к технологическому газу, определяется по формуле:

$$
Q_{\text {shell }}=U_{\text {shell }} A_{\text {shell }}\left(T_{\text {shell }}-T_{\mathrm{g}}\right),
$$

где $A_{\text {shell }}$ - площадь поверхности, через которую передаётся тепло от стенки аппарата к газу, $\mathrm{M}^{2} ; U_{\text {shell }}-$ коэффициент теплопередачи от стенки к газу, $\mathrm{B} \mathrm{T} /\left(\mathrm{M}^{2} \cdot \mathrm{K}\right) ; T_{\text {shell }}-$ температура стенки аппарата десублимации, $\mathrm{K}$.

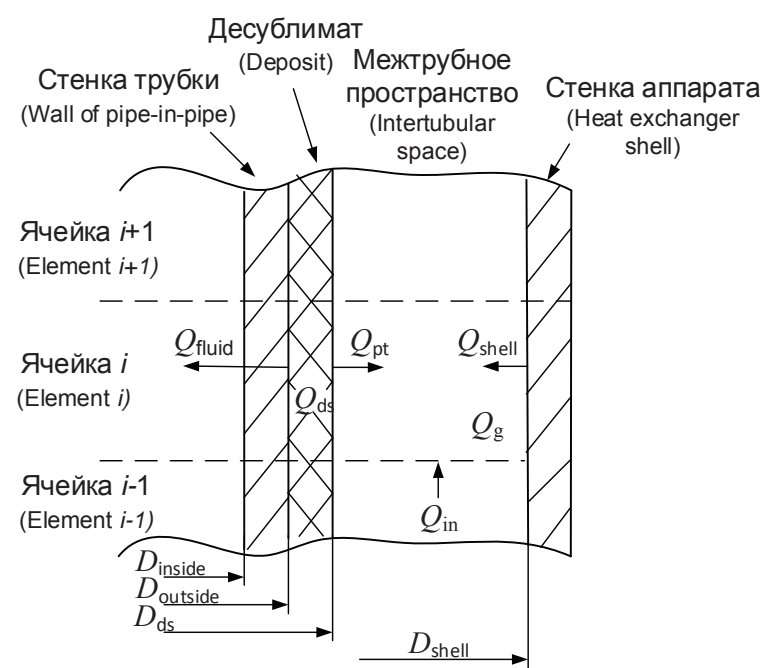

Pис. 4. Направления движения тепловых потоков внутри ячей$\kappa и: Q_{\mathrm{pt}}-$ тепловой поток от десублилата $\kappa$ теплоносителю, кДж; $Q_{\mathrm{g}}-$ количество тепла, передаваемое технологическому газу, кДж; $Q_{\text {shell }}$ - тепловой поток от стенки ап-

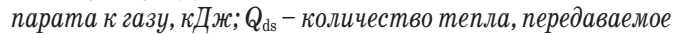
слою десублимата, кДж; $Q_{\text {in }}$ - количество тепла, приноси-

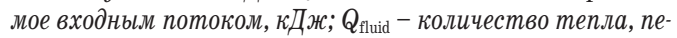
редаваемое хладагенту, кДж; $D_{\text {inside }}-$ внутренний диаметр трубки, м; $D_{\text {outside }}$ - наружный диаметр трубки, м; $D_{\mathrm{ds}}$ - диаметр трубки со слоем десублимата, м

Fig. 4. Heat transfer directions in the element: $Q_{\mathrm{pt}}$ is the amount of heat transfer from deposit to gas mixture, $k J ; Q_{\mathrm{g}}$ is the amount of heat energy of the gas mixture in the element, $k J$; $Q_{\text {shell }}$ is the amount of heat transfer from the exchanger shell to the gas mixture, $k J ; Q_{\mathrm{ds}}$ is the amount of heat energy of the deposit, $k J ; Q_{\text {in }}$ is the amount of heat transfer from the inlet gas mixture flow, $k J ; Q_{\text {fluid }}$ is heat transfer from the deposition to fluid, $k J ; D_{\text {inside }}$ is the inside diameter of the outer pipe, $m$; $D_{\text {outside }}$ is the outside diameter of the outer pipe, $m$; $D_{\mathrm{ds}}$ is the outside diameter of the outer pipe with the deposition layer, $m$

Количество тепла, передаваемое от входного потока технологического газа $Q_{\text {in }}$ в ячейку, определяется через разность энтальпий:

$$
Q_{\text {in }}=v_{\text {in }} A_{\text {in }} C_{\text {in }}\left(s_{\mathrm{g}}\left(T_{\mathrm{g}}\right)-s_{\text {in }}\left(T_{\text {in }}\right)\right) \text {, }
$$

где $s_{\mathrm{g}}$ - энтальпия состояния технологического газа в ячейке, кДж/моль; $s_{\text {in }}$ - энтальпия состояния технологического газа во входном потоке, кДж/моль.

Фазовый переход газообразного гексафторида в твёрдый является экзотермическим процессом. Тепловой эффект от превращения определятся разностью энтальпий веществ в этих агрегатных состояниях: 


$$
Q_{\mathrm{pt} i}=m_{\mathrm{ds} i}\left(s_{\mathrm{UF}_{6} \mathrm{~g}}\left(T_{\mathrm{g}}\right)-S_{\mathrm{UF}_{6} \mathrm{ds}}\left(T_{\mathrm{ds} i}\right)\right) \text {, }
$$

где $s_{\mathrm{UF}_{6} \mathrm{~g}}$ - энтальпия газообразного $\mathrm{UF}_{6}$, кДж/моль; $s_{\mathrm{UF}_{6} \mathrm{ds}}-$ энтальпия твёрдого $\mathrm{UF}_{6}$ на $i$ секции, кДж/моль; $m_{\mathrm{ds} i}-$ масса нового десублимата на $i$ секции, моль.

Количество тепла, передаваемого от теплоносителя к слою десублимата, движущегося в секции, рассчитывается:

$$
Q_{\text {fluid } i}=U_{\text {fluid } i} A_{\text {sur } i}\left(T_{\text {fluid } i}-T_{\text {ds } i}\right) \text {, }
$$

где $A_{\text {sur } i}$ - площадь поверхности, через которую передаётся тепло в $i$ секции хладагенту, $\mathrm{m}^{2} ; U_{\text {fluid } i}-$ коэффициент теплопередачи в $i$ секции от слоя десублимата к хладагенту, Вт/( $\left.\mathrm{M}^{2} \cdot \mathrm{K}\right), T_{\text {fluid } i}-$ температура хладагента в $i$ секции, $K$.

\section{Массообменные процессы}

Аппарат десублимации предназначен для получения твёрдого гексафторида урана из технологического газа. Данный процесс является фазовым переходом первого рода газ-твёрдое. Согласно диаграмме состояния гексафторида урана, данный процесс происходит при температуре ниже 329,5 К и атмосферном давлении. В таблице приведены температуры фазовых переходов для остальных компонентов технологического газа при атмосферном давлении [9-11].

таблица. Температура фазового перехода компонентов газа

Table. $\quad$ Phase transformation temperature of gas mixture components

\begin{tabular}{|c|c|c|}
\hline $\begin{array}{c}\text { Kомпонент газа } \\
\text { Gas mixture } \\
\text { component }\end{array}$ & $\begin{array}{c}\text { Температура } \\
\text { конденсации, } \mathrm{K} \\
\text { Condensation, } \mathrm{K}\end{array}$ & $\begin{array}{c}\text { Температура } \\
\text { затвердевания, } \mathrm{K} \\
\text { Solidification, } \mathrm{K}\end{array}$ \\
\hline $\mathrm{HF}$ & 292,9 & 189,9 \\
\hline $\mathrm{N}_{2}$ & 77,2 & 63,14 \\
\hline $\mathrm{O}_{2}$ & 90 & 53 \\
\hline $\mathrm{F}_{2}$ & 88 & 55 \\
\hline
\end{tabular}

Как видно из табл. 1, при температуре в 329,5 К ни один из компонентов технологического газа не подвержен процессу десублимации. Таким образом, в аппарате будет проходить десублимация только гексафторида урана. При моделировании используются физические свойства $\mathrm{UF}_{6}$, описанные в литературе [12-14]. Существует ряд работ по моделированию процесса десублимации ГФУ в осадительных ёмкостях и поверхностных аппаратах $[15,16]$. Однако используемые в работах методы описания процесса десублимации сложно применимы для проточных аппаратов.

При описании массообменных процессов рассматривались два режима работы: захолаживание и отпаривание. Процесс захолаживания характеризуется длительным поддержанием разности температур хладагента и технологического газа на стенках трубчатки. Скорость нарастания десублимата напрямую связана с концентрацией веществ в технологическом газе. Масса десублимата на $i$ секции определяется следующим уравнением [17]:

$$
\frac{m_{\mathrm{ds} i}}{d t}=K A_{\mathrm{ds}} M_{\mathrm{UF}_{6}} \frac{d\left(C_{\mathrm{UF}_{6}}\left(T_{\mathrm{g}}\right)-C_{\mathrm{eq}}\left(T_{\mathrm{ds} i}\right)\right)}{d t},
$$

где $K$ - коэффициент скорости массопередачи, $\mathrm{m} / \mathrm{c} ; A_{\mathrm{ds} i}-$ площадь взаимодействия поверхности трубы $i$ секции и газа, $\mathbf{m}^{2} ; M_{\text {Uf6 }}$ - молярная масса $\mathrm{UF}_{6}$, кг/моль; $C_{\text {еq }}$ - равновесная концентрация, моль $/ \mathrm{m}^{3} ; C_{\mathrm{UF6}}-$ концентрация газообразного гексафторида в ячейке, моль $/ \mathrm{m}^{3}$;

Процесс нарастания и уплотнения десублимата является достаточно сложным для математического описания. Существуют исследования по выявлению закономерностей влияния длительности процесса десублимации и температуры десублимации на плотность получаемого продукта $[18,19]$. Однако в данной работе при разработке математической модели принято допущение, что фазовый переход происходит только в поверхностном слое, непосредственно контактирующем с технологическим газом. При этом в процессе нарастания слоя формируется равномерно плотный продукт. По мере нарастания слоя десублимата температура поверхностного слоя будет приближаться к температуре технологического газа, а следовательно, будет замедляться скорость десублимации. Процесс десублимации прекращается, когда вследствие увеличения термодинамического сопротивления слоя десублимата температура поверхностного слоя десублимата, контактирующего с газовой фазой, сравнивается с температурой, соответствующей значению наступления фазового равновесия при текущей концентрации ГФУ в составе газа.

Отпаривание характеризуется резкой сменой температуры на поверхности трубчатки за счёт смены хладагента на теплоноситель. Замеры длительности переходных процессов при смене режима работы аппарата на производстве показали, что они занимают несколько секунд. Это даёт основания принять допущение, что нагревание поверхности происходит мгновенно, равномерно и полностью. Следовательно, весь наросший слой десублимата осыпается в транспортные ёмкости в результате сублимации его на границе раздела с трубчаткой за установленный на заводе интервал сброса.

\section{Проверка адекватности математической модели}

Целевое назначение разработанной математической модели аппарата десублимации - определение длительности интервала захолаживания трубчатки, обеспечивающего накопление на её поверхности слоя десублимата заданной толщины. Проверка адекватности математической модели проводилась путем сравнения масс накапливаемого десублимата в транспортной емкости, полученных на действующем производстве и в результате вычислительных экспериментов. В качестве входных значений при проведении вычислительных экспериментов использовались производственные данные за сравниваемый период.

Сравнение производственных данных и результатов вычислительных экспериментов по накопле- 
нию десублимата в транспортной емкости при одинаковых входных значениях технологических переменных представлены на рис. 5. Производственные данные соответствуют показаниям весоизмерительного устройства.

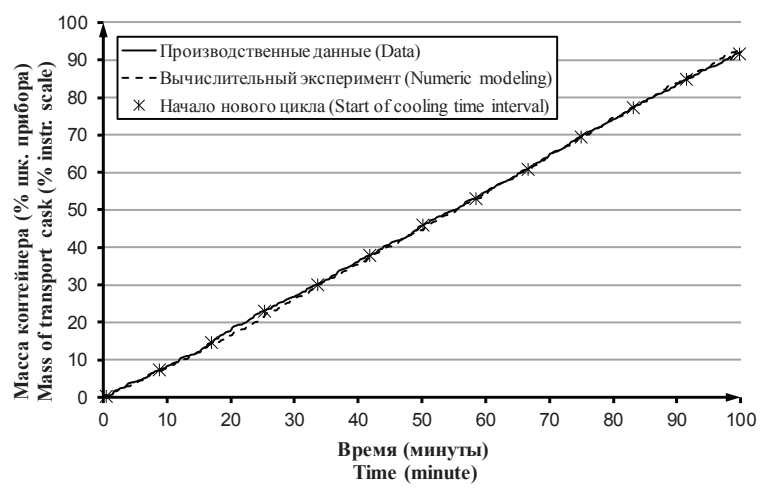

Pис. 5. График производственных данных и результатов вычи слительных экспериментов по накоплению десублимата в транспортной емкости

Fig. 5. Graph of the production data and the numeric modeling results of the deposit accumulating in the transport cask

Таким образом, были промоделированы 19 интервалов захолаживания за разные даты работы производства. Среднеквадратичная погрешность результатов моделирования, приведённая к массе накопленного десублимата за один интервал захолаживания, составила $9,3 \%$. Расчёт погрешности проводился по формуле:

$$
s=\frac{\sigma}{\Delta P} \cdot 100 \%=\sqrt{\frac{\left(P_{\text {data }}-P_{\text {model }}\right)^{2}}{n-1}} \cdot \frac{100 \%}{\Delta P},
$$

где $\Delta P$ - величина приращения массы десублимата на трубчатке за интервал захолаживания, \% шк. прибора; $P_{\text {data }}$ - масса накопленного десублимата по показаниям весоизмерительного устройства, \% шк. прибора; $P_{\text {model }}$ - масса накопленного десублимата по результатам вычислительных экспериментов, \% шк. прибора.

Полученные результаты позволяют судить о возможности применения разработанной математической модели для моделирования работы аппарата десублимации при различных интервалах захолаживания.

\section{Уравнение расчёта времени захолаживания}

Для получения уравнения расчёта интервала захолаживания использовались методы статистического анализа [20, 21]. При составлении плана проведения полного факторного эксперимента в качестве факторов использовались следующие технологические переменные:

- задаваемая оператором толщина слоя десублимата $\Delta p$, мм;

- температура хладагента $T_{\text {fluid }}, \mathrm{K}$;

- объёмный расход технологического газа на входе в аппарат $Q_{\mathrm{vg}}, \mathrm{M}^{3} /$ ч;
- объёмная доля ГФУ в составе поступающего технологического газа $C_{\mathrm{v} \mathrm{UF} 6}$, об. \% .

В результате проведения вычислительных экспериментов на разработанной математической модели аппарата десублимации производства ГФУ получены регрессионные уравнения расчёта величины интервала захолаживания. Приведённая среднеквадратичная погрешность полученных уравнений регрессии 1-го и 2-го порядка составила 12 и $0,7 \%$ соответственно. Уравнение 2-го порядка точнее описывает данные вычислительных экспериментов. Итоговое уравнение для расчёта величины интервала захолаживания $t_{\mathrm{ct}}$, обеспечивающего накопление заданной толщины слоя десублимата, имеет вид:

$$
\begin{gathered}
t_{\mathrm{ct}}=10^{3}-6,1 Q_{\mathrm{vg}}-87,7 C_{\mathrm{v} \mathrm{UF}_{6}}+19,3 T_{\text {fluid }}+ \\
+5,4 \cdot 10^{2} \Delta p+6,23 \cdot 10^{-2} \cdot Q_{\mathrm{v} \mathrm{g}} C_{\mathrm{v} \mathrm{UF}}- \\
8,7 \cdot 10^{-3} Q_{\mathrm{vg}} T_{\text {fluid }}-1,9 Q_{\mathrm{vg}} \Delta p- \\
-0,6 \cdot C_{\mathrm{v} \mathrm{UF}_{6}} T_{\mathrm{cl}}-33,9 C_{\mathrm{v} \mathrm{UF}_{6}} \Delta p+8,4 T_{\text {fluid }} \Delta p+ \\
8,2 \cdot 10^{-3} Q_{\mathrm{vg}}^{2}+1,1 C_{\mathrm{v} \mathrm{UF}_{6}}^{2}+0,3 T_{\text {fluid }}^{2}-1,2 \cdot 10^{2} \Delta p^{2} .
\end{gathered}
$$

\section{Заключение}

Сложность задачи по управлению аппаратами десублимации производства ГФУ связанна с переменными расходом, составом технологического газа и температурой охлаждающей жидкости. Каждая из этих технологических переменных оказывает влияние на процесс десублимации. Для реализации системы управления аппаратом десублимации длительность интервала захолаживания используется в качестве управляющего воздействия, что обеспечивает накопление заданного слоя десублимата на поверхности трубчатки.

Исследования процесса накопления твёрдого ГФУ на трубчатке проводились на разработанной математической модели аппарата десублимации, описывающей основные физико-химические процессы и закономерности, протекающие внутри аппарата.

Результаты вычислительных экспериментов на математической модели использованы для построения регрессионной модели процесса захолаживания, позволяющей вычислять длительность накопления десублимата как функцию регистрируемых на производстве технологических переменных и заданной оператором толщины слоя десублимата с погрешностью 9,3\%. В итоге предложен алгоритм расчёта интервала захолаживания аппарата десублимации производства ГФУ, который обеспечит равномерность заполнения транспортных ёмкостей в пределах установленного на заводе допуска в $1 \%$.

Работа поддержана Министерством образования и науки Российской Федерации, базовая часть «Наука», проект 2.5760.2017/8.9. 


\section{СПИСОК ЛИТЕРАТУРЫ}

1. Thomas S. Russia's Nuclear Export Programme // Energy Policy. 2018. - V. 121. - P. 236-247.

2. Вильнина А.В., Ливенцов С.Н. Адаптивный алгоритм управления узлом десублимации гексафторида урана // Известия Томского политехнического университета. - 2008. - Т. 312. № 5. - C. 133-136.

3. Орлов А.А., Малюгин Р.В. Анализ способов получения гексафторида урана, очистки его от примесей и заполнения в транспортные емкости // Современные наукоемкие технологии. Региональное приложении. - 2014. - № 3. - С. 89-98.

4. Development of a desublimator design / I.Y. Rusakov, P.B. Molokov, A.S. Buinovskii, V.L. Sofronov, V.N. Brendakov // Chemical and Petroleum Engineering. - 2017. - V. 53. - № 1-2. P. 89-93.

5. Uniform deposition of uranium hexafluoride(UF6): Standardized mass deposits and controlled isotopic ratios using a thermal fluorination method / B.K. McNamara, M.J. O'Hara, A.M. Casella, J.C. Carter, R.S. Addleman, P.J. MacFarlan // Talanta. 2016. - V. 154. - P. 219-227.

6. Байдали С.А., Дядик В.Ф., Юрков А.С. Математическая модель производства гексафторида урана // Известия Томского политехнического университета. - 2009. - Т. 315 - № 2. C. $84-90$.

7. Николаев А.В., Криницын Н.С., Дядик В.Ф. Математическая модель десублиматора производства гексафторида урана // Известие вузов. Физика. - 2015. - Т. 58. - № 12-3. - С. 97-103.

8. Беккер В.Ф. Моделирование химико-технологических объектов. - М.: ИНФРА-М, 2014. - 142 c.

9. Patnaik P. Handbook of inorganic chemicals. - USA: McGrawHill, 2002. - $1046 \mathrm{p}$.

10. Weinstock B., Crist, R.H. The vapor pressure of uranium hexafluoride // The Journal of Chemical Physics. - 1948. - V. 16. № 5. - P. 436-441.

11. Физико-математическое моделирование десублимации фтористого водорода из газовой смеси на стенки конденсатора / И.М. Васенин, С.М. Губанов, М.И. Дурновцев, А.Ю. Крайнов, М.В. Чуканов // Вестник томского государственного универси- тета. Математика и механика. - 2014. - Т. 31. - № 5. C. $76-82$.

12. Oliver G.D., Milton H.T., Grisard J.W. The Vapor Pressure and Critical Constants of Uranium Hexafluoride // Journal of the American Chemical Society. - 1953. - V. 75. - № 12. P. 2827-2829.

13. Zhang J. Analysis on holdup during processing UF6 // Journal of radioanalytical and nuclear chemistry. - 2014. - V. 299. - № 1. P. 517-522.

14. Определение коэффициента теплопроводности твердой фазы гексафторида урана / В.В. Раев, А.В. Сапрыгин, А.П. Цедилкин, К.В. Новокшонов // Фундаментальные проблемы современного материаловедения. - 2011. - Т. 8. - № 4. - С. 125-130.

15. Gubanov S.M., Krainov A.Y., Mazur R.L. Theoretical and experimental modeling of the cooling of uranium hexafluoride vapor desublimation tanks // Theoretical Foundations of Chemical Engineering. - 2016. - V. 50. - № 5. - P. 766-775.

16. Intensification of evaporation of uranium hexafluoride / A.M. Belyntsev, G.S. Sergeev, 0.B. Gromov, V.G. Frolov, V.F. Kholin // Theoretical Foundations of Chemical Engineering. 2013. - V. 47. - № 4. - P. 499-504.

17. Горелик А.Г., Амитин А.В. Десублимация в химической промышленности. - М.: Химия, 1986. - 272 с.

18. Study of frost properties correlating with frost formation types / Y. Hayashi, A. Aoki, S. Adachi, K. Hori // Journal of Heat Transfer. - 1977. - V. 99 (2). - P. 239-245.

19. Lee J., Lee K.-S. The behavior of frost layer growth under conditions favorable for desublimation / International Journal of Heat and Mass Transfer. - 2018. - V. 120. - P. 259-266.

20. George E.P., Hunter J.S., Hunter W.G. Statistics for Experimenters: Design, Innovation, and Discovery. $2^{\text {nd }}$ ed. - USA: John Wiley \& Sons, 2005. - $664 \mathrm{p}$.

21. Дядик В.Ф., Байдали С.А., Байдали Т.А. Статистические методы контроля и управления. - Томск: Изд-во Томского политехнического университета, 2011. - $144 \mathrm{c.}$

Поступила 17.12.2018 2.

\section{Информация об авторах}

Николаев А.В., инженер научно-образовательной лаборатории электроники и автоматики физических установок Инженерной школы ядерных технологий Национального исследовательского Томского политехнического университета.

Криницын H.C., кандидат технических наук, заведующий лабораторией промышленной робототехники Инженерной школы информационных технологий и робототехники Национального исследовательского Томского политехнического университета.

Дядик В.Ф., кандидат технических наук, доцент отделения ядерно-топливного цикла Инженерной школы ядерных технологий Национального исследовательского Томского политехнического университета. 
UDC: $661.879 .1 .04-52$

\title{
ALGORITHM OF AUTOMATIC CONTROL OF URANIUM HEXAFLUORIDE PRODUCTION DEPOSITION
}

\author{
Alexander V. Nikolaev', \\ niav@tpu.ru \\ Nikolay S. Krinitsyn', \\ kns@tpu.ru \\ Valery F. Dyadik', \\ dyadik@tpu.ru \\ 'National Research Tomsk Polytechnic University, \\ 30, Lenin Avenue, Tomsk, 634050, Russia.
}

The relevance of the research is caused by the requirement for uniform filling of transport casks with uranium hexafluoride and for solution to stop filling it according to the degree of its fill up. This requirement can be achieved by the commissioning of an automated control system for deposition apparatus.

The main aim of the research is to develop the algorithm for calculating the cooling interval of the deposition apparatus tube bundle, which ensures the accumulation of the given deposit layer thickness in an automated mode.

Object of the research is uranium hexafluoride deposition apparatus.

Methods: mathematical modeling of technological processes; full-factor experiments on the mathematical model; regression analysis; statistical analysis; field experiments on the deposition apparatus for uranium hexafluoride production.

Results. Duration of cooling interval is used as a control action for implementation of stabilization system for a given deposit layer thickness on the cooled element (tube bundle). The mathematical model of the deposition apparatus is used to conduct numerical experiments and to find an analytical dependence for calculating the duration of the cooling interval. The mathematical model describes the heat exchange and mass transfer occurring in the apparatus. The adequacy of the model is confirmed by comparing the results of mathematical modeling and production data. The conclusion about the adequacy of the model is made by the magnitude of the reduced standard error of prediction by the model of production data. The equation for calculating the cooling interval was obtained using the developed mathematical model as a result of a full factorial experiment. The equation allows calculating the required duration of the cooling interval for the tube bundle, during which the operator-specified deposit layer will be accumulated.

Key words:

Deposition, uranium hexafluoride, heat transfer, mass transfer, numeric modeling

The research was supported by the Ministry of Education and Science of the Russian Federation, basic part «Science», project $2.5760 .2017 / 8.9$.

\section{REFERENCES}

1. Thomas S. Russia's Nuclear Export Programme. Energy Policy, 2018, vol. 121, pp. 236-247.

2. Vilnina A.V., Liventsov S.N. The adaptive algorithm of control over the unit of desublimation of uranium hexafluoride. Bulletin of the Tomsk Polytechnic University, 2008, vol. 312, no. 5, pp. 133-136. In Rus.

3. Orlov A.A., Malugin R.V. The analysis of uranium hexafluoride production, its cleaning from impurities and filling in transport capacities. Modern highway technologies. Regional appendix, 2014, no. 3, pp. 89-98. In Rus.

4. Rusakov I.Y., Molokov P.B., Buinovskii A.S., Sofronov V.L., Brendakov V.N. Development of a desublimator design. Chemical and Petroleum Engineering, 2017, vol. 53, no. 1-2, pp. 89-93.

5. McNamara B.K., O'Hara M.J., Casella A.M., Carter J.C., Addleman R.S., MacFarlan P.J. Uniform deposition of uranium hexafluoride (UF6): Standardized mass deposits and controlled isotopic ratios using a thermal fluorination method. Talanta, 2016, vol. 154, pp. 219-227.

6. Bajdali S., Diadik V., Yurkov A. Mathematical Model of the Production of Uranium Hexafluoride. Bulletin of Tomsk Polytechnic University, 2009, vol. 315, no. 2, pp. 84-90. In Rus.

7. Nikolaev A.V., Krinitsyn N.S., Dyadik V. F. Mathematical model desublimator uranium hexafluoride production. Russian Physics Journal, 2015, vol. 58, no. 12-3, pp. 97-103. In Rus.
8. Bekker V.F. Modelirovanie khimiko-tekhnologicheskih obektov [Modeling of chemical-technological objects]. Moscow, INFRA-M Publ., 2014. 142 p.

9. Patnaik P. Handbook of inorganic chemicals. USA, McGraw-Hill Publ., 2002. 1046 p.

10. Weinstock B., Crist R.H. The vapor pressure of uranium hexafluoride. The Journal of Chemical Physics, 1948, vol. 16, no. 5, pp. $436-441$.

11. Vasenin I.M., Gubanov S.M., Durnovtsev M.I., Kraynov A.Y. Chukanov M.V. Physico-mathematical modeling of fluoride hydrogen desublimation from gas mixtures onto walls of a condenser. Tomsk state university journal of mathematics and mechanics, 2014, vol. 31, no. 5, pp. 76-82. In Rus.

12. Oliver G.D., Milton H.T., Grisard J.W. The Vapor Pressure and Critical Constants of Uranium Hexafluoride. Journal of the American Chemical Society, 1953, vol. 75, no. 12, pp. 2827-2829.

13. Zhang J. Analysis on holdup during processing UF6. Journal of radioanalytical and nuclear chemistry, 2014, vol. 299, no. 1, pp. 517-522.

14. Raev V.V., Saprygin A.V., Tsedilkin A.P., Novokshonov K.V. Determination of thermal conductivity of the solid phase of uranium hexafluoride. Basic Problems of Material Science, 2011, vol. 8, no. 4, pp. 125-130. In Rus.

15. Gubanov S.M., Krainov A.Y., Mazur R.L. Theoretical and experimental modeling of the cooling of uranium hexafluoride vapor 
desublimation tanks. Theoretical Foundations of Chemical Engineering, 2016, vol. 50, no. 5, pp. 766-775.

16. Belyntsev A.M., Sergeev G.S., Gromov 0.B., Frolov V.G., Kholin V.F. Intensification of evaporation of uranium hexafluoride. Theoretical Foundations of Chemical Engineering, 2013, vol. 47, no. 4, pp. 499-504.

17. Gorelik A.G., Amitin A.V. Desublimatsiya v khimicheskoy promyshlennosti [Desublimation in the chemical industry]. Moscow, Khimiya Publ., 1986. 272 p.

18. Hayashi Y., Aoki A., Adachi S., Hori K. Study of frost properties correlating with frost formation types. Journal of Heat Transfer, 1977, vol. 99 (2), pp. 239-245.
19. Lee J., Lee K.-S. The behavior of frost layer growth under conditions favorable for desublimation. International Journal of Heat and Mass Transfer, 2018, vol. 120, pp. 259-266.

20. George E.P., Hunter J.S., Hunter W.G. Statistics for Experimenters: Design, Innovation, and Discovery. $2^{\text {nd }}$ ed. USA, John Wiley \& Sons Publ., 2005. 664 p.

21. Diadik V.F., Baidali S.A., Baidali T.A. Statisticheskie metody kontrolya i upravleniya [Statistical methods of control and management]. Tomsk, Tomsk Polytechnic University Publ., 2011. $144 \mathrm{p}$.

Received: 17 December 2018.

\section{Information about the authors}

Alexander V. Nikolaev, engineer, National Research Tomsk Polytechnic University.

Nikolay S. Krinitsyn, Cand. Sc., head of research laboratory for industrial robotics, National Research Tomsk Polytechnic University.

Valery F. Dyadik, Cand. Sc., assistant professor, National Research Tomsk Polytechnic University. 